\title{
Open
}

\section{A Randomized Placebo-Controlled Phase IIb Trial of A3309, A Bile Acid Transporter Inhibitor, for Chronic Idiopathic Constipation}

William D. Chey, MD, AGAF, FACG, FACP ${ }^{1}$, Michael Camilleri, MD, AGAF, FACG, FACP², Lin Chang, MD, AGAF³, Leif Rikner, MSc ${ }^{4}$ and Hans Graffner, MD, PhD4

OBJECTIVES: $\quad$ A3309 is a minimally absorbed ileal bile acid (BA) transporter (IBAT) inhibitor. We conducted an 8-week, multicenter, randomized, double-blind, placebo-controlled, parallel group, phase IIb study, which evaluated A3309 in patients with chronic idiopathic constipation (CIC).

METHODS: $\quad$ Patients with CIC (modified Rome III criteria and $<3$ complete (CSBM) spontaneous bowel movements (SBMs)/week during the 2-week baseline) were randomized to 5,10 , or $15 \mathrm{mg} \mathrm{A3309}$ or placebo once daily. The primary end point was change in SBM number during week 1 compared with baseline. Other bowel and abdominal symptoms were assessed as secondary end points. Serum $7 \alpha \mathrm{C} 4$ and lipids were evaluated as biomarkers of BA synthesis/loss.

RESULTS: $\quad$ In all, 190 patients (mean 48 years, 90\% female) were randomized. Mean increase (95\% confidence interval) in SBM for week 1 were $1.7(0.7-2.8)$ for placebo vs. 2.5 (1.5-3.5), 4.0 (2.9-5.0), and 5.4 (4.4-6.4) for $5 \mathrm{mg}, 10 \mathrm{mg}(P<0.002)$, and $15 \mathrm{mg}(P<0.001)$ A3309, respectively. Increased stool frequency was maintained over 8 weeks. Time to first SBM and CSBM were significantly reduced in the 10- and 15-mg A3309 groups compared with placebo. Straining and bloating decreased with A3309 compared with placebo $(P<0.05)$. Increased $7 \alpha C 4$ and reduced low-density lipoprotein cholesterol with A3309 suggested increased BA synthesis and BA loss. The most common adverse events (AEs) were abdominal pain and diarrhea, which occurred most commonly in the 15-mg A3309 group. No drug-related serious AEs were observed.

CONCLUSIONS: A3309 increased stool frequency and improved constipation-related symptoms in CIC; effects were maintained over 8 weeks of treatment.

SUPPLEMENTARY MATERIAL is linked to the online version of the paper at http://www.nature.com/ajg

Am J Gastroenterol 2011; 106:1803-1812; doi:10.1038/ajg.2011.162; published online 24 May 2011

\section{INTRODUCTION}

Chronic idiopathic constipation (CIC) is a common gastrointestinal (GI) condition that affects quality of life and health-care expenditures (1). It has been estimated that $12-19 \%$ (2) of the general population experiences CIC. Constipation is more often reported by females and those over 65 years of age (2). The symptoms of CIC are diverse and include infrequent bowel movements (BMs), hard stools, excessive straining, bloating, and abdominal discomfort/pain (3). Though a number of over-the-counter and prescription medications are available, approximately half of patients with CIC are not satisfied with their current treatment
(4). As such, there remains an unmet need for medical therapies and approaches addressing novel physiological mechanisms in patients with CIC.

The pathophysiology of CIC remains incompletely defined. There is evidence to suggest that modulation of endogenous bile acid (BA) homeostasis might offer a novel therapeutic approach to the treatment of CIC. There are several examples demonstrating that changes in intracolonic BA concentrations alter bowel function: ileal resection results in diarrhea (5), and changes in diurnal rates of BA synthesis and colonic BA concentration have been identified in constipated children (6) and in adults with slow

${ }^{1}$ Department of Medicine, University of Michigan Health System, Ann Arbor, Michigan, USA; ${ }^{2}$ Mayo Clinic, Rochester, Minnesota, USA; ${ }^{3}$ David Geffen School of Medicine at UCLA, Los Angeles, California, USA; ${ }^{4}$ Albireo, Goteborg, Sweden. Correspondence: William D. Chey, MD, AGAF, FACG, FACP, Department of Medicine, University of Michigan Health System, 3912 Taubman Center, SPC 5362, Ann Arbor, Michigan 48109-5362, USA. E-mail: wchey@med.umich.edu Received 27 January 2011; accepted 12 April 2011 
transit constipation and constipation-predominant irritable bowel syndrome (IBS-C) $(7,8)$. Conversely, decreasing free BA concentrations in the colon through the use of the BA sequestrant cholestyramine induces constipation (5), and another BA sequestrant, colesevelam, retards accelerated colonic transit in patients with IBS-D (9).

Inhibitors of the ileal BA transporter (IBAT; syn ASBTapical sodium-dependent BA transporter) reduce the active ileal reabsorption of $\mathrm{BA}$, resulting in an increased concentration of BA entering the colon, thereby stimulating colonic motility and secretion (10). BAs induce propulsive contractions in the human colon (11), secretion through activation of adenylate cyclase (12), increased mucosal permeability (13), and inhibition of apical $\mathrm{Cl}^{-}$/ $\mathrm{OH}^{-}$exchange (14). These effects on colonic motility and secretion may offer clinical benefits in patients with CIC. A3309 is a selective inhibitor of the IBAT, which has been shown to reduce meat-induced constipation in the dog (15). In humans, A3309 accelerated colonic transit in a multiple ascending dose, phase $\mathrm{Ib}$ study in patients with CIC (16).

The current phase IIb study in patients with CIC was performed to assess the short-term efficacy and safety of orally administered A3309 at 5, 10, and $15 \mathrm{mg}$ doses, compared with placebo, once daily for 8 weeks.

\section{METHODS}

\section{Study design: randomization and protocol}

A randomized, double-blind, placebo-controlled study was conducted at 45 sites across the United States between November 2009 (first signed informed consent) and August 2010 (last patient visit). The study was conducted in accordance with the Declaration of Helsinki and with Good Clinical Practice guidelines. Written informed consent was obtained from all patients before participation. Central or local Ethical Review Boards approved the study protocol and the informed consent form. The study was registered on ClinicalTrial.Gov [NCT01007123]. Treatment allocation and concealment were conducted by a computerized random-number generator and numbered containers with active and placebo capsules of identical appearance. The randomization list was centrally held by an independent Contract Research Organization, all randomized patients and study center and contract research organization personnel were blinded to study treatment allocation until data were locked and analyzed.

After informed consent was obtained, patients entered an initial screening period of up to 28 days during which routine blood tests, urinalysis, and pregnancy testing were obtained. Where appropriate, patients were taken off of laxatives and other prohibited medications. Specific washout periods (3- or 10-day periods based on duration of action) were used for prohibited medications. Bulking agents and stool softeners were allowed if the patient was taking a stable dose for at least 30 days before the start of the screening period. The use of rescue medication (bisacodyl suppository) was permitted following consultation with the investigator if the patient had $>72 \mathrm{~h}$ without a BM. Patients meeting the inclusion and exclusion criteria entered a 14-day pretreatment baseline period. Patients eligible to continue were randomized to receive a 5-, 10-, or 15-mg tablet of A3309 or placebo orally once daily before breakfast for 8 weeks. During the 14 days before randomization and during the treatment period, patients reported daily bowel and abdominal symptoms using an Interactive Voice Response System. Study visits occurred during the screening period, at randomization (day 0), during treatment (day 28), at end of treatment (day 56 ), and 14 days post-treatment (day 70).

\section{Study medication}

Pharmacokinetic studies show that orally administered A3309 leads to minimal systemic exposure and that systemically available drug is highly protein bound (>99\%) (15).

\section{Study participants}

Eligible patients were men or non-pregnant women, 20-80 years of age, who met modified Rome III criteria for functional constipation (17), with a diagnosis of $>3$ months and with symptom onset at least 6 months before diagnosis. Study participants were required to have a baseline $\mathrm{BM}$ frequency of $<3$ complete spontaneous BMs (CSBMs) per week. In addition, $\geq 2$ of the following characteristics that are included in the Rome III criteria for functional constipation were required during the 14 days before randomization: (1) straining during $\geq 25 \%$ of BMs, (2) sensation of incomplete evacuation in $\geq 25 \%$ of BMs, (3) lumpy or hard stools in $\geq 25 \%$ of BMs, or (4) sensation of blockage/anorectal obstruction in $\geq 25 \%$ of BMs. Eligible patients had a body mass index of $>18.0$ and $<35 \mathrm{~kg} / \mathrm{m}^{2}$. Patients were excluded if they had been previously diagnosed with constipation-predominant IBS, had abdominal pain as a predominant symptom, or had drug-induced constipation. Also, patients were excluded if they reported the need for manual maneuvers (digital insertion into the rectum, perianal pressure, or vaginal splinting) or frequent use of enemas to evacuate stool. Other exclusion criteria included known rectal prolapse, dyssynergic defecation (per patient history and clinical examination), previous intestinal resection, neurological cause of constipation, and use of drugs with known effects on GI motility or stool consistency. Patients reporting loose or liquid stool consistency by a Bristol Stool Form Scale score of 6 or 7 (unless within $24 \mathrm{~h}$ of having taken a laxative) at least once during the screening period were excluded (18). Patients with persistent constipation symptoms despite a stable dosage of bulking agents for $>4$ weeks were eligible.

All study participants were required to meet the colorectal cancer screening requirements of the American Gastroenterological Association Guidelines (19). Women of child-bearing age were required to have a negative serum pregnancy test; those who were sexually active were required to use an accepted form of birth control during their participation in the study.

\section{Outcome measures and assessments}

The primary end point of the study was the change from baseline in frequency of weekly spontaneous bowel movements (SBMs) at week 1 . Secondary efficacy end points included weekly assessment of SBMs and CSBMs, daily assessments of other bowel- 
related complaints (i.e., stool consistency and straining), and abdominal symptoms (i.e., pain, discomfort, and bloating). The time to onset of clinical efficacy was evaluated by "time to first SBM" and "percentage of patients having an SBM within $24 \mathrm{~h}$ of first administration of study drug." Similar assessments for CSBM were also performed. In addition, we chose to look at the stool frequency responder end point included in a recent US Food and Drug Administration guidance document (20); "CSBM stool frequency responder" was defined as a patient who had an increase of $\geq 1$ CSBM per week over baseline for at least 4 of the 8 weeks of the treatment period.

\section{Daily symptom assessments}

Assessments were recorded by phone using Interactive Voice Response System. Each day, patients recorded the time study medication was taken and the number and time of each BM. The characteristics of each BM were assessed using the 7-point Bristol Stool Form Scale (18). The severity of straining was graded using a 5 -point ordinal scale $(1=$ not at all, $2=$ a little bit, $3=$ more straining than not, $4=$ a great deal, and $5=$ an extreme amount). SBM was defined as a BM in which no laxative, enema, or suppository was used in the preceding $24 \mathrm{~h}$, and a CSBM was defined as an SBM, which was associated with a feeling of complete bowel emptying. Each day, patients also recorded the severity of bloating, pain, and discomfort using 5 -point scales $(1=$ none, $2=$ mild, $3=$ moderate, $4=$ severe, and $5=$ very severe) and any use of rescue medication.

\section{Surrogate markers of hepatic synthesis and GI loss of BAs}

Mechanistic effects of A3309 on hepatic BA synthesis rates were evaluated using plasma C4 ( $7 \alpha$-hydroxy-4-cholesten-3-one) (21) and on plasma lipids (total cholesterol, low-density lipoprotein (LDL) cholesterol, high-density lipoprotein (HDL) cholesterol, and triglycerides) using a high-performance liquid chromatography method.

\section{Safety assessment}

The intensity of adverse events (AEs) was reported by patients as mild, moderate, or severe. Separate from AE intensity, patients were defined as suffering from a serious $\mathrm{AE}$ (SAE) if the $\mathrm{AE}$ resulted in death, was deemed life threatening, led to long-term disability, or required or prolonged hospitalization for treatment. The site investigator assessed all patient-reported AEs and determined their relationship to study treatment. Safety evaluations included physical examinations, electrocardiogram, vital signs measurements, and standard laboratory tests (clinical chemistry, hematology, fat-soluble vitamins [A, D, E], and coagulation).

\section{Statistical methods and data analysis}

Patients were randomized in a 1:1:1:1 ratio to the treatment groups. The sample size for this study was selected to provide $80 \%$ power to detect a difference of $2.5 \mathrm{SBMs}$ per week between an active treatment group and the placebo group using a two-sided $t$-test at $\alpha=0.05$ significance without adjustment for multiplicity. This calculation was based on the assumption that treatment groups have unequal variances (estimated active s.d. $=4.7$, estimated pla- cebo s.d. $=2.6$ ). Thirty-eight patients per treatment group were required to achieve the desired power under the stated assumptions. To allow for a withdrawal rate of $15 \%, 45$ patients per group (180 patients total) were required.

All changes from baseline end points were analyzed with a one-way analysis of covariance, with a fixed effect for treatment group and the baseline value of SBMs used as a covariate. Within the analysis of covariance, each dose of A3309 was compared with placebo in a pairwise manner. The time to the first SBM and the first CSBM were analyzed using a one-way analysis of variance, with a fixed effect for treatment group. The binary response end point "CSBM stool frequency responder" was analyzed with Fisher's exact test comparing each A3309 dose with placebo.

An observed-cases approach to missing data was applied (i.e., a patient's missing values were not imputed or carried forward). All $P$ values are based on two-sided tests and given that this was a phase IIb dose-range finding study, the reported $P$ values were not adjusted for multiple comparisons. Patients discontinuing treatment were defined as treatment failures for the responder analyses.

Efficacy analyses are reported for the ITT (intent to treat) population defined as all randomized patients who received at least one dose of study medication and had any post-baseline patient diary data (BM or GI symptoms) collected $(n=190)$.

Evaluations of serum $\mathrm{C} 4$ and lipids, which reflect pharmacodynamic effects of A3309, were performed on the completer per protocol population $(n=121)$ defined as all ITT patients who completed the study and were at least $80 \%$ compliant with study medication and patient reports during the treatment period.

Safety results are reported for the safety population $(n=189)$ defined as all randomized patients who received at least one dose of study treatment and for whom follow-up safety data are available. Assessments of safety were performed evaluating number/ proportions of patients experiencing SAEs/AEs and with an analysis of any changes from baseline in laboratory safety tests, electrocardiogram, and vital signs.

\section{RESULTS}

\section{Patient flow and follow-up}

Of 448 CIC patients screened, 190 were randomized and 161 completed the study (Figure 1). At baseline, the demographic parameters were similar between the treatment groups (Table $\mathbf{1}$ ). Patients had a mean age of 48.1 years (range 20-79), and were predominantly female (90\%) and white (78\%). The mean number of SBMs and CSBMs per week at screening was 2.82 and 0.40 , respectively. Full baseline characteristics of our study population can be found in Supplementary Appendix Table 1 online.

\section{Bowel function}

Stool frequency. At week 1 of the double-blind treatment phase (primary efficacy end point), patients treated with 10 or $15 \mathrm{mg}$ of A3309 reported a significantly $(P<0.002$ and $P<0.001$, respectively) greater change from baseline in the number of SBMs compared with placebo; Figure 2 shows a clear dose-response with mean (95\% confidence interval) increases of $2.5(1.5-3.5, \mathrm{NS})$, 


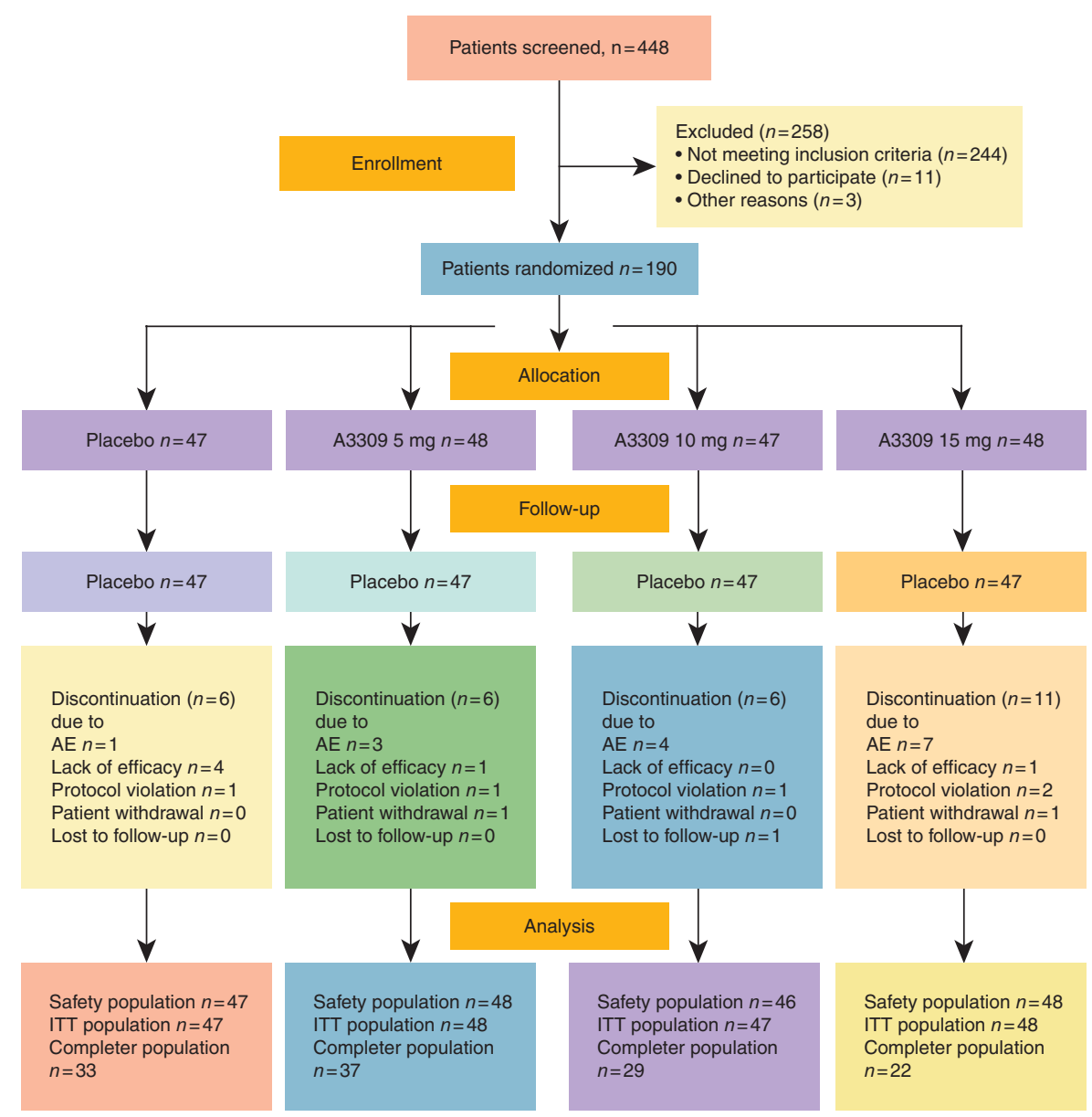

Figure 1. Patient flow diagram. Completer PP (per protocol) population=all intention to treat (ITT) patients who completed the study and were at least $80 \%$ compliant with study medication and patient reports during the treatment period. AE, adverse event.

4.0 (2.9-5.0, $P<0.002)$, and $5.4(4.4-6.4 ; P<0.001)$ SBMs for the A3309 dose levels of 5, 10, and $15 \mathrm{mg}$, compared with an increase of 1.7 (0.7-2.8) SBMs for placebo. The beneficial effect of A3309 on weekly SBM (Figure 3a) and CSBM (Figure 3b) frequency was maintained over placebo for the 8-week treatment period.

A3309 at doses of 10 and $15 \mathrm{mg}$ significantly reduced the time to first SBM relative to placebo ( $P=0.033$ and 0.039 , respectively); an SBM occurred (mean) 12 and $7 \mathrm{~h}$ after first dose of A3309 10 and $15 \mathrm{mg}$, respectively, compared with (mean) $27 \mathrm{~h}$ for placebo. A significantly higher proportion of patients reported an SBM within $24 \mathrm{~h}$ of taking their first dose of A3309 at a dose of 10 (74\%) or $15 \mathrm{mg}(75 \%)$ compared with placebo (45\%) $(P=0.012$ for both). When considering time to first CSBM or the proportion who reported a CSBM within $24 \mathrm{~h}$ of dosing, significant differences were again observed with the two higher doses of A3309 compared with placebo ( $P \leq 0.02$ for all comparisons).

CSBM stool frequency responder rates were also significantly improved with all three A3309 dose levels compared with placebo; $58 \%$ (25/43), $64 \%$ (27/42), $75 \%(30 / 40)$ for the 5,10 , and $15 \mathrm{mg}$ groups compared with placebo $(33 \%, 14 / 42)(P=0.030$, $0.008,<0.001$, respectively).
Stool consistency and straining. Patients treated with 10 and $15 \mathrm{mg}$ doses of A3309 reported significant improvements in stool consistency and significant decreases in straining at all treatment weeks ( $P<0.001$ for all comparisons) (Figure $4 \mathbf{a}$ and $\mathbf{b}$ ).

\section{Abdominal symptoms}

Bloating, pain, and discomfort. The mean change from baseline in overall bloating across the 8 -week treatment period improved in patients receiving the 15-mg dose of A3309 $(P=0.048)$ (Figure 4c). There were no differences in effects on abdominal pain or discomfort between the A3309 groups and placebo.

\section{Rescue medication use}

There were no significant differences in the use of rescue medication between active treatment groups and placebo at any of the study intervals.

\section{Surrogate markers of hepatic BA synthesis and GI loss}

Plasma C4 increased in all of the active A3309 dose levels with mean ( \pm s.e.m.) changes from baseline being $1.2( \pm 4.2)$ for placebo, $14.9( \pm 4.0)$ for the 5-mg group, $21.1( \pm 4.4)$ for the 10-mg group, 


\begin{tabular}{|c|c|c|c|c|c|}
\hline & \multirow[t]{2}{*}{ Placebo } & \multicolumn{3}{|c|}{ A3309 } & \multirow[t]{2}{*}{ Overall } \\
\hline & & $5 \mathrm{mg}$ & $10 \mathrm{mg}$ & $15 \mathrm{mg}$ & \\
\hline$N$ & 47 & 48 & 47 & 48 & 190 \\
\hline \multicolumn{6}{|l|}{ Age, years } \\
\hline \multicolumn{6}{|l|}{ Age group } \\
\hline$<60$ years & $33(70.2)$ & 40 (83.3) & $39(83.0)$ & $40(83.3)$ & $152(80.0)$ \\
\hline$\geq 60$ years & $14(29.8)$ & $8(16.7)$ & $8(17.0)$ & $8(16.7)$ & $38(20.0)$ \\
\hline \multicolumn{6}{|l|}{ Gender } \\
\hline White & $35(74.5)$ & $41(85.4)$ & $36(76.6)$ & $36(75.0)$ & 148 (77.9) \\
\hline Black/African American & $12(25.5)$ & $7(14.6)$ & $10(21.3)$ & $12(25.0)$ & $41(21.6)$ \\
\hline \multicolumn{6}{|l|}{$B M I, k g / m^{2}$} \\
\hline Mean & 26.87 & 26.09 & 27.09 & 27.71 & 26.94 \\
\hline s.d. & 4.330 & 4.327 & 3.728 & 3.841 & 4.076 \\
\hline
\end{tabular}

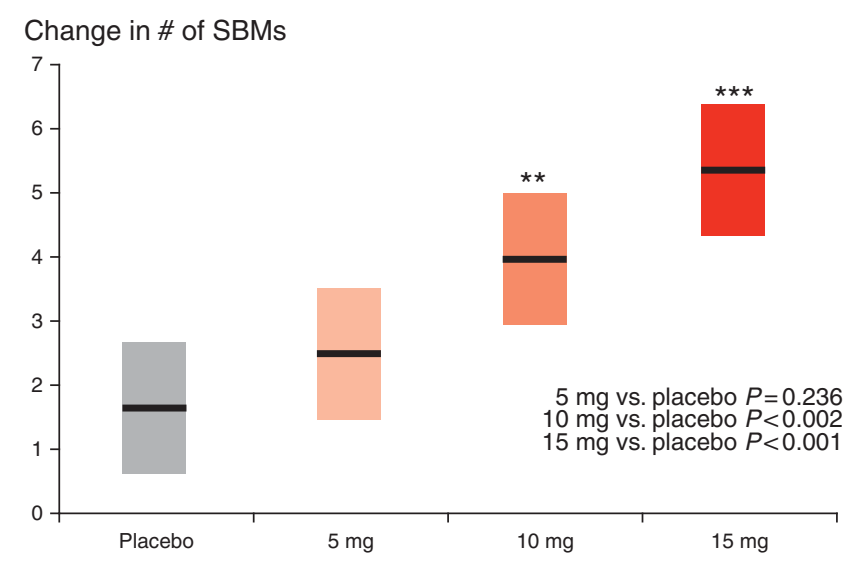

Least squares mean (95\% confidence interval) - ITT population

Figure 2. Change from baseline in weekly spontaneous bowel movement (SBM) frequency for treatment week 1 . ITT, intention to treat.

and $12.9( \pm 5.1)$ for the 15 -mg group $(P=0.020,0.002$, and 0.082 , respectively). Baseline total cholesterol levels were $>200 \mathrm{mg} / \mathrm{dl}$ in $43 \%$ of the patients; the mean ( \pm s.e.m.) baseline total plasma cholesterol level was $195( \pm 5.2) \mathrm{mg} / \mathrm{dl}$ (ITT population). Significant decreases in total cholesterol were observed for the 10- and 15-mg dose levels ( $P=0.014$ and $P=0.027$, respectively). Compared with baseline, LDL cholesterol and the LDL/HDL ratio each decreased while on treatment with A3309 (Table 2). Neither A3309 nor placebo caused significant changes in plasma HDL cholesterol or triglycerides.

\section{Safety assessment}

AEs were reported in 102 of the 189 patients (54\%); 14 patients (7\%) had events of severe intensity. The main difference between the groups was that five patients in the 15-mg A3309 group had abdominal cramping/pain and/or diarrhea of severe intensity. Treatment-emergent SEs occurred in 44, 46, 62, and 65\% in the placebo, 5, 10, 15mg A3309 groups, respectively. The most common AEs were of GI origin including abdominal pain $(0,10,11$, and $27 \%)$ and diarrhea $(2,8,6$, and $13 \%)$.

In total, 29 patients (15\%) discontinued study medication (placebo: $12.8 \%, 5 \mathrm{mg}$ A3309: $12.5 \%, 10 \mathrm{mg}$ A3309: 12.8\%, and $15 \mathrm{mg}$ A3309: 22.9\%). Table 3 outlines the treatment-emergent AEs and discontinuations during the study. The rate of AEs and discontinuations was greater in the 15-mg A3309 group compared with the 5- or 10-mg A3309 or placebo groups. Discontinuations due to GI AEs are outlined in Table 3.

Three SAEs were reported, one in the placebo group (shoulder pain), one in the 5-mg A3309 group (colonoscopy verified bleeding colonic diverticulum 2 weeks after the end of treatment), and one in the 10-mg A3309 group (breast carcinoma diagnosed on day 5 of treatment). None of the SAEs were considered related to the study drug. No deaths occurred during the study.

There were no differences in the incidence of clinically significant changes in laboratory parameters, vital signs, or other safety parameters. 
a

$\leadsto$ Placebo $-5 \mathrm{mg} \leftarrow 10 \mathrm{mg} \multimap 15 \mathrm{mg}$

\# of SBMs/week

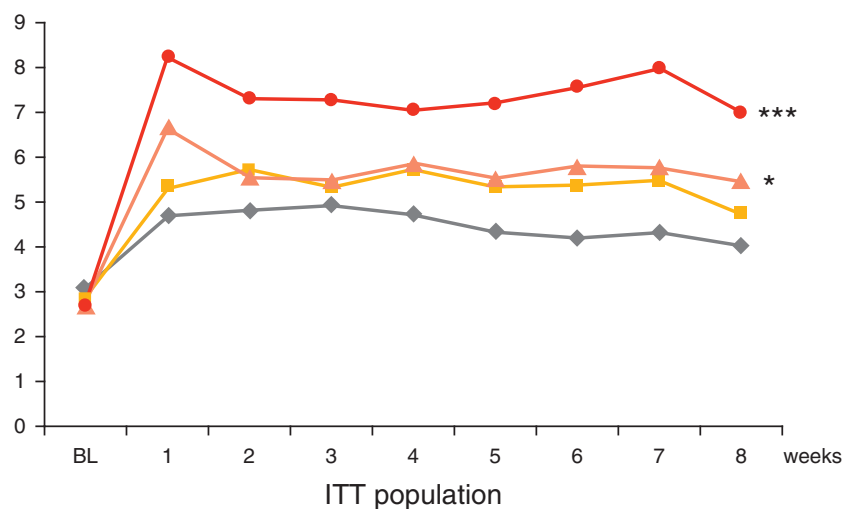

b

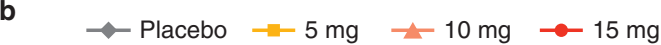

\# of CSBMs/week

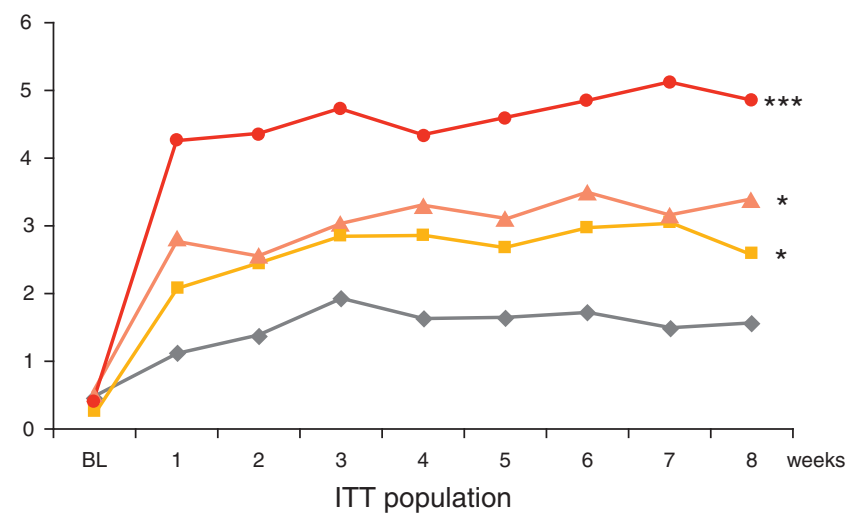

Figure 3. Effect of A3309 and placebo on weekly stool frequency. Mean weekly SBM (a) and CSBM (b) frequency. Significance values shown are for the overall change from baseline between A3309 and placebo. ${ }^{* *} P<0.001,{ }^{*} P<0.05$. CSBM, complete spontaneous bowel movement; ITT, intention to treat; SBM, spontaneous bowel movement.

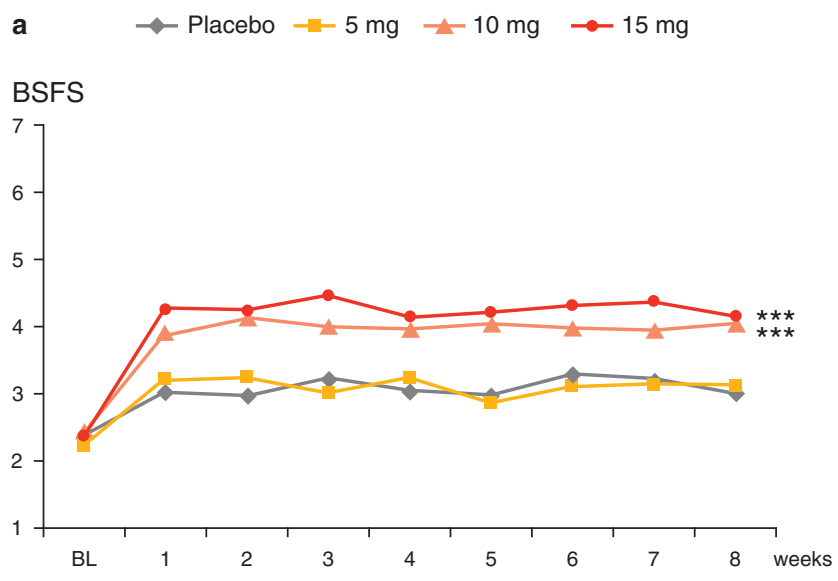

b $\neg$ Placebo $-5 \mathrm{mg} \multimap 10 \mathrm{mg} \longrightarrow 15 \mathrm{mg}$

Degree of straining

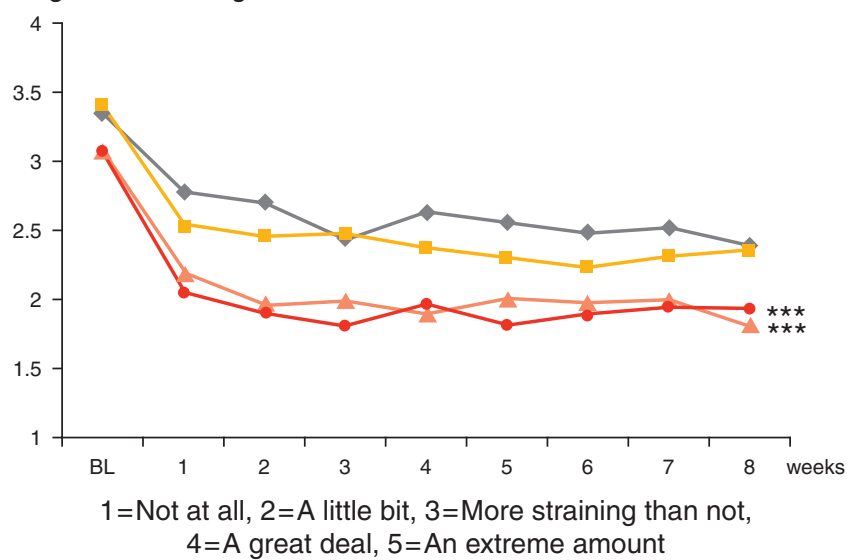

ITT population

Degree of bloating

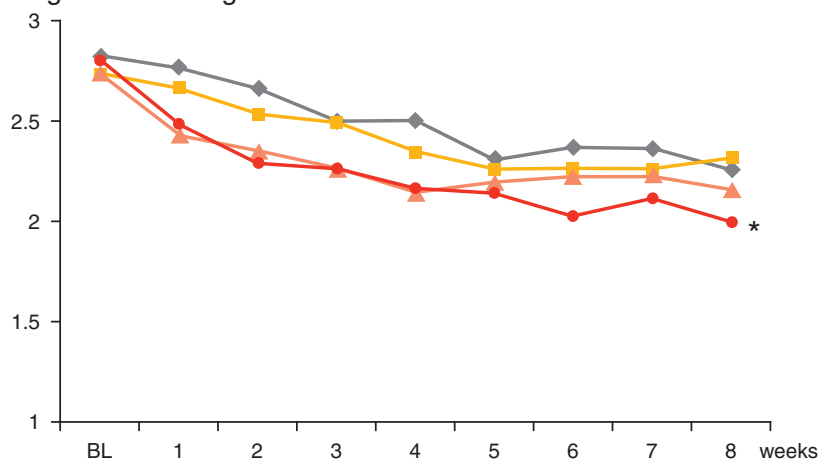

$1=$ None, $2=$ Mild, $3=$ Moderate, $4=$ Severe, $5=$ Very severe

ITT population

Figure 4. Effect of A3309 and placebo on weekly stool consistency, straining, and bloating. Changes in stool consistency (a), straining (b), and bloating (c) over the treatment period. Significance values shown are for the overall change from baseline between A3309 and placebo. ${ }^{* * *} P<0.001,{ }^{*} P<0.05$. BSFS, Bristol Stool Form Scale (7-graded scale, see text for explanations); ITT, intention to treat. 


\section{DISCUSSION}

This randomized, placebo-controlled trial evaluated the efficacy and safety of three doses of the novel IBAT inhibitor, A3309 in patients with CIC. Compared with placebo, A3309 significantly increased SBMs during week 1 (the primary end point) and each subsequent week of the 8-week treatment period. A3309 also significantly increased weekly CSBMs, an end point that includes the patient's qualitative assessment of completeness of a bowel movement and arguably provides an even more rigorous assessment of stool frequency than SBM. A3309 led to significant improve-

\begin{tabular}{|c|c|c|c|c|}
\hline & \multirow[t]{2}{*}{ Placebo } & \multicolumn{3}{|c|}{ A3309 } \\
\hline & & $5 \mathrm{mg}$ & $10 \mathrm{mg}$ & $15 \mathrm{mg}$ \\
\hline$N$ & 32 & 37 & 29 & 22 \\
\hline \multicolumn{5}{|l|}{$L D L$} \\
\hline Mean change & 2.6 & -2.4 & $-15.6^{* * *}$ & $-11.1^{* *}$ \\
\hline s.d. & 17.30 & 15.61 & 18.23 & 22.90 \\
\hline \multicolumn{5}{|l|}{$L D L / H D L$} \\
\hline Mean change & -0.024 & -0.111 & $-0.287^{* *}$ & $-0.221^{*}$ \\
\hline s.d. & 0.333 & 0.405 & 0.318 & 0.393 \\
\hline
\end{tabular}

HDL, high-density lipoprotein; ITT, intention to treat; LDL, low-density lipoprotein. Completer PP (per protocol) population (all ITT patients who completed the study and were at least $80 \%$ compliant with study medication and patient reports during the treatment period).

${ }^{* * *} P<0.001,{ }^{* *} P=0.01,{ }^{*} P<0.05$. ments in other constipation-associated symptoms (stool consistency, straining, and bloating). There was also benefit for the rigorous CSBM stool frequency responder definition (an increase of $\geq 1$ CSBM per week over baseline for at least 4 of the 8 weeks of the treatment period), with all doses of A3309 compared with placebo. The effects of A3309 on the frequency and consistency of BMs occurred within the first week of drug administration and were maintained over the full 8 weeks of treatment. Roughly three quarters of the patients in the 10- and 15-mg A3309 groups reported passing an SBM within $24 \mathrm{~h}$ of initial drug dosing. Importantly, there was no diminution of the clinical benefits of A3309 over time, suggesting that there was no development of tolerance.

It has long been known that BAs are physiological promoters of colonic secretion and motility (10). Reduced synthesis and/or colonic concentrations of specific BAs have been associated with the development of constipation (6-8). Therefore, recent work has investigated the potential role of raising colonic BA concentration (by direct supplementation or through prevention of ileal reabsorption) as a novel target for new drug development in patients with CIC $(8,16)$. BAs are mainly absorbed in the ileum by a specific BA transporter or IBAT. The IBAT is an integral brush border membrane glycoprotein that co-transports sodium and BAs. Available evidence suggests that the IBAT is a major regulator of the BA pool in animals and humans. BA reabsorption from the intestine is very efficient; $95 \%$ of the secreted BAs are reabsorbed and delivered back to the liver completing the process of enterohepatic circulation (22).

IBAT inhibitors prevent the reabsorption of BAs from the ileum and consequently, their return to the liver. The liver compensates

Table 3. Discontinuations and related treatment-emergent gastrointestinal adverse events (TEAEs)

\begin{tabular}{|c|c|c|c|c|}
\hline & Placebo $(N=46)$ & A3309 $5 \mathrm{mg}(N=48)$ & A3309 $10 \mathrm{mg}(N=47)$ & A3309 $15 \mathrm{mg}(N=48)$ \\
\hline Treatment discontinuations & $6(12.8)$ & $6(12.5)$ & $6(12.8)$ & $11(22.9)$ \\
\hline \multicolumn{5}{|l|}{ Gastrointestinal disorders } \\
\hline Total & 5 (10.9) & $12(25.0)$ & $11(23.4)$ & 19 (39.6) \\
\hline Mild & $2(4.3)$ & $6(12.5)$ & $5(10.6)$ & $6(12.5)$ \\
\hline Severe & $1(2.2)$ & $1(2.1)$ & $2(4.3)$ & $5(10.4)$ \\
\hline Abdominal pain & 0 & $5(10.4)$ & $5(10.6)^{a}$ & $13(27.1)^{\text {aаaа }}$ \\
\hline Diarrhea & $1(2.2)$ & $4(8.3)$ & $3(6.4)^{a}$ & $6(12.5)^{\text {aаaа }}$ \\
\hline Abdominal distension & $1(2.2)$ & $4(8.3)$ & $2(4.3)^{a}$ & $3(6.3)^{\text {aa }}$ \\
\hline Abdominal pain upper & $1(2.2)$ & $1(2.1)$ & 0 & $1(2.1)$ \\
\hline
\end{tabular}


for the decrease in BAs by upregulating cholesterol $7 \alpha$-hydroxylase, the rate-limiting enzyme for BA synthesis. This results in increased levels of $7 \alpha$-hydroxy-4-cholesten-3-one (C4), an intermediate in BA synthesis. In addition, lower hepatic cholesterol levels and increased number of LDL receptors in the liver occur leading to reduced plasma LDL cholesterol $(23,24)$. These concepts were confirmed in our study where A3309 but not placebo resulted in increased $\mathrm{C} 4$ values and decreased plasma total and LDL cholesterol levels, consistent with increased BA synthesis due to inhibition of IBAT. A3309's cholesterol-lowering effect may be an incremental attribute of this treatment that may prove useful in patients with CIC.

The improvements to constipation symptoms observed with A3309 are likely the consequence of increased delivery of BAs to the colon leading to increased fluid secretion and motility and resultant acceleration of transit. This suggestion is supported by a recent single center, randomized, double-blind dose escalation study in 30 patients with CIC, which found that A3309 significantly accelerated colon transit measured by radio-opaque markers and increased stool frequency compared with placebo (16).

Contrary to the clear benefit of A3309 for constipation-associated symptoms, there was no statistically significant difference in abdominal pain or discomfort ratings between the A3309 and placebo groups. The interpretation of these findings may be confounded by low baseline scores for abdominal pain and discomfort (floor effect) likely resulting from the exclusion of patients with a prior history of IBS or abdominal pain or discomfort as a predominant complaint. Further evaluation of A3309 in patients with IBSC should be considered to more definitively evaluate the impact of this drug on abdominal pain and abdominal discomfort.

A3309 was generally well tolerated and no SAEs related to the study drug were reported. There was a dose-related trend in the number of AEs and in the percentage of patients discontinuing treatment. Because A3309 has a very low systemic exposure (resulting in estimated plasma concentrations in the picomolar range), it is reasonable to speculate that AEs, which were largely GI in origin, were the consequence of the increased concentration of colonic BAs $(11,25)$.

Based upon data from this phase IIb dose-range study, it appears that the 10-mg dose of A3309 provided the best balance of efficacy and safety in patients with CIC. Patients on the 15-mg dose level had more AEs of abdominal pain and diarrhea. It is conceivable that this may reflect a direct effect of the higher dose of A3309 on motility, rather than a secondary effect on transit arising from increased colonic secretion. Concentrations of $>5 \mathrm{mM}$ of BAs infused directly into the human rectum and sigmoid stimulate colonic phasic contractions (11); in addition, Bampton et al. (26) observed HAPCs in the colon with administration of $1 \mathrm{mM}$ chenodexoycholate into the rectum of healthy volunteers. Such concentrations of 1-5 mM BAs are seldom achieved in the absence of ileal resection (27).

Despite the chronic nature of CIC, many patients use medical therapy intermittently; the speed of onset of A3309 is also potentially beneficial as it significantly shortened the time to first SBM and CSBM. Treatment with A3309 may therefore be suited for individualized treatment, either on a fixed schedule or "on demand."
Further studies specifically designed to address these potential uses for A3309 in CIC patients are eagerly awaited.

Forty-three percent of CIC patients enrolled in this study had baseline total plasma cholesterol values above the desirable cutoff level of $200 \mathrm{mg} / \mathrm{dl}$. A3309 treatment induced a decrease in LDL cholesterol and in the important predictor of cardiovascular disease-the LDL/HDL ratio-(14\% decreases in the 10-mg dose group for both variables). The beneficial effects on lipid profile are a unique feature of A3309 among current medications and those in the pipeline for treatment of CIC. This improvement in serum lipids might provide incremental benefits to a subset of patients with CIC.

When considering our results, several additional issues merit discussion. As is common in drug trials of this size, a large number of investigative sites were included and patient-reported outcomes were captured using an Interactive Voice Response System or with electronic handheld devices. Studies that rely upon electronic data capture might bias the randomized population toward a younger, more affluent demographic. What effect, if any, this could have on our results is unknown. Patients with CIC entered into this study were required to be passing fewer than 3 CSBMs per week. This may limit the generalizability of our data to the entire population of CIC patients. Acknowledging this point, the decision for this entry requirement was largely predicated upon current regulatory recommendations, which support stool frequency as the primary outcome for trials evaluating therapies for CIC. Further, though weekly CSBM rate was used to determine study eligibility, weekly SBM rate after the first week of therapy served as the primary outcome measure. This primary outcome was chosen after consultation with the US Food and Drug Administration. In addition to meeting the primary outcome, the two higher doses of A3309 provided robust and significant benefits to the rate of weekly SBMs and CSBMs for the entire 8-week treatment period. One further point, which merits consideration, is that our study population likely included a subset of patients with dyssynergic defecation. The study protocol called for the exclusion of patients with a history of dyssynergic defecation or in whom the history and physical examination was felt to indicate the presence of this type of constipation. We acknowledge that this is an imperfect way of identifying patients with dyssynergic defecation and that the inclusion of such patients could have affected the study results. However, we would point out that nearly all other recently performed trials evaluating novel drug therapies for chronic constipation have handled the issue of dyssynergic defecation in a similar manner $(28,29)$. In addition, randomization would be expected to level the effect of the inclusion of such patients on results yielded by the study groups. We would also argue that the practice used in this protocol closely mimics clinical practice where diagnostic testing for dyssynergic defecation is typically reserved for patients who have failed multiple laxative therapies as recommended by the American Gastroenterological Association (30) and the Rome Foundation (31).

In conclusion, the results of this placebo-controlled, dose-range, phase IIb study in CIC patients demonstrated that the IBAT inhibitor A3309 significantly improved BM frequency, stool consistency, and constipation symptoms. The 10-mg dose of A3309 provided 
the best balance of efficacy and safety. Further large clinical trials evaluating the efficacy of A3309 in patients with CIC and IBS-C are warranted.

\section{ACKNOWLEDGMENTS}

We thank the ACCESS Study team investigators and nurses for their participation in this study.

\section{CONFLICT OF INTEREST}

Guarantor of the article: William D. Chey, MD, AGAF, FACG, FACP.

Specific author contributions: Full access to all the data in the study and took responsibility for the integrity of the data and the accuracy of the data analyses and wrote the first draft of the manuscript: William D. Chey and Hans Graffner. No professional writing assistance was utilized in the preparation of this manuscript. All authors contributed to study concept and design and data analyses, drafted and reviewed the manuscript revisions, and approved the final draft for submission. The statistical analysis of the entire data sets has been independently analyzed by a biostatistician not employed by Albireo and with no links to the institution performing the study. Financial support: Albireo funded this study and provided A3309. Potential competing interests: Rikner and Graffner are employees of Albireo. Chey is a consultant for Albireo. Camilleri receives a research grant from Albireo, and serves as a consultant for Albireo with payment of consultant fee to Mayo Clinic. Chang serves as a consultant for Albireo.

\section{Study Highlights}

\section{WHAT IS CURRENT KNOWLEDGE}

Alterations in bile acid metabolism and decreased colonic bile acid concentrations have been implicated in the pathogenesis of constipation.

Bile acids induce colonic secretion and motility and accelerate colon transit.

The ileal bile acid transporter (IBAT) is responsible for the reuptake of bile acids from the terminal ileum.

The IBAT inhibitor A3309 acts locally to decrease bile acid reuptake and has been shown to accelerate colon transit in patients with chronic constipation.

\section{WHAT IS NEW HERE}

In a methodologically rigorous phase IIb trial, A3309 provided rapid and sustained improvement in stool frequency and other constipation-related symptoms compared with placebo.

A3309 increased bile acid synthesis and reduced plasma low-density lipoprotein (LDL) cholesterol and the LDL/highdensity lipoprotein (HDL) ratio.

The most common adverse events with $\mathrm{A} 3309$ were abdominal pain and diarrhea. These adverse events appeared to be dose dependent.

In a dose-response evaluation, the 10-mg A3309 dose provided a good balance of efficacy and safety and may be a suitable therapeutic alternative for patients with chronic constipation.

\section{REFERENCES}

1. Talley NJ. Functional gastrointestinal disorders as a public health problem. Neurogastroenterol Motil 2008;20 (Suppl 1): 121-9.

2. Higgins PD, Johansen JF. Epidemiology of constipation in North America: a systematic review. Am J Gastroenterol 2004;99:750-9.

3. Pare P, Ferrazzi S, Thompson WG et al. An epidemiological survey of constipation in Canada: definitions, rates, demographics, and predictors of health care seeking. Am J Gastroenterol 2001;96:3130-7.

4. Johanson JF, Kralstein J. Chronic constipation: a survey of the patient perspective. Aliment Pharmacol Ther 2007;25:599-608.

5. Hofmann AF, Poley JR. Cholestyramine treatment of diarrhea associated with ileal resection. N Engl J Med 1969;281:397-402.

6. Hofmann AF, Loening-Baucke V, Lavine JE et al. Altered bile acid metabolism in childhood functional constipation: inactivation of secretory bile acids by sulfation in a subset of patients. J Pediatr Gastroenterol Nutr 2008:47:598-606.

7. Abrahamsson H, Ostlund-Lindqvist AM, Nilsson R et al. Altered bile acid metabolism in patients with constipation-predominant irritable bowel syndrome and functional constipation. Scand J Gastroenterol 2008;43:1483-8.

8. Rao AS, Wong B, Camilleri M et al. Chenodeoxycholate in females with irritable bowel syndrome-constipation: a pharmacodynamic and pharmacogenetic analysis. Gastroenterology 2010;139:1549-58.

9. Odunsi-Shiyanbade ST, Camilleri M, McKinzie S et al. Effects of chenodeoxycholate and a bile acid sequestrant, colesevelam, on intestinal transit and bowel function. Clin Gastroenterol Hepatol 2010;8:159-65.

10. Bajor A, Gillberg P-G, Abrahamsson H. Bile acids: short and long term effects in the intestine. Scand J Gastroenterol 2010;45:645-64.

11. Kirwan WO, Smith AN, Mitchell WD et al. Bile acids and colonic motility in the rabbit and the human. Gut 1975;16:894-902.

12. Conley DR, Coyne MJ, Bonorris GG et al. Bile acid stimulation of colonic adenylate cyclase and secretion in the rabbit. Am J Dig Dis 1976;21:453-8.

13. Chadwick VS, Gaginella TS, Carlson GL et al. Effect of molecular structure on bile acid-induced alterations in absorptive function, permeability, and morphology in the perfused rabbit colon. J Lab Clin Med 1979;94:661-74.

14. Alrefai WA, Saksena S, Tyagi S et al. Taurodeoxycholate modulates apical $\mathrm{Cl}-/ \mathrm{OH}-$ exchange activity in Caco2 cells. Dig Dis Sci 2007;52:1270-8.

15. Gillberg PG, Dahlström M, Starke I et al. The IBAT inhibition by A3309-a potential mechanism for the treatment of constipation. Gastroenterology 2010;5 (Suppl 1): 224.

16. Simren M, Abrahamsson H, Bajor A et al. The IBAT inhibitor A3309-a promising treatment option for patients with chronic idiopathic constipation. Gastroenterology 2010;5 (Suppl 1): 223.

17. Longstreth GF, Thompson WG, Chey WD et al. Functional bowel disorders. Gastroenterology 2006;130:1480-91.

18. O'Donell LJ, Virjee J, Heaton KW. Detection of pseudodiarrhoea by simple clinical assessment of intestinal transit rate. Br Med J 1990;300:439-40.

19. Rex D, Johnson DA, Anderson JC et al. American college of gastroenterology guidelines for colorectal cancer screening 2008. Am J Gastroenterol 2009; 104:739-50.

20. Food and Drug Administration [Internet]. Guidance for Industry: Irritable Bowel Syndrome - Clinical Evaluation of Products for Treatment [updated 4 March 2010; cited 27 January 2011]. Available at: http://www. fda.gov/downloads/Drugs/GuidanceComplianceRegulatoryInformation/ Guidances/UCM205269.pdf.

21. Gälman C, Angelin B, Rudling M. Bile acid synthesis in humans has a rapid diurnal variation that is asynchronous with cholesterol synthesis. Gastroenterology 2005;129:1445-53.

22. Angelin B, Eriksson M, Rudling M. Bile acids and lipoprotein metabolism: a renaissance for bile acids in the post-statin era? Curr Opin Lipidol 1999;10:269-74.

23. Bertolotti M, Zambianchi L, Carulli L et al. Influence of newly synthesized cholesterol on bile acid synthesis during chronic inhibition of bile acid absorption. Hepatology 2003;38:939-46.

24. Naoumova RP, O’Neill FH, Dunn S et al. Effect of inhibiting HMG-CoA reductase on $7 \alpha$-hydroxy-4-cholesten-3-one, a marker of bile acid synthesis: contrasting findings in patients with and without prior up-regulation of the latter pathway. Eur J Clin Invest 1999;29:404-12.

25. Edwards CA, Brown S, Baxter AJ et al. Effect of bile acids on anorectal function in man. Gut 1989;30:383-6. 
26. Bampton PA, Dinning PG, Kennedy ML et al. The proximal colonic motor response to rectal mechanical and chemical stimulation. Am J Physiol Gastrointest Liver Physiol 2002;282:G443-9.

27. Mitchell WD, Findlay JM, Prescott RJ et al. Bile acids in the diarrhoea of ileal resection. Gut 1973;14:348-53.

28. Johanson JF, Wald A, Tougas G et al. Effect of tegaserod in chronic constipation: a randomized, double-blind, controlled trial. Clin Gastroenterol Hepatol 2004;2:796-805.

29. Lembo AJ, Kurtz CB, Macdougall JE et al. Efficacy of linaclotide for patients with chronic constipation. Gastroenterology 2010;138: $886-95$.
30. Locke GR III, Pemberton JH, Phillips SF. American gastroenterological association medical position statement: guidelines on constipation. Gastroenterology 2000;119:1761-6.

31. Spiller RC, Thompson WG. Bowel disorders. Am J Gastroenterol 2010;105:775-85.

(c) This work is licensed under the Creative Commons

OMTERIGHTS RESERVED Attribution-NonCommercial-No Derivative Works 3.0

Unported License. To view a copy of this license, visit http://creativecommons.org/licenses/by-nc-nd/3.0/ 